\title{
Pacientes com câncer: enfrentamento, rede social e apoio social
}

\author{
Jeanny Joana Rodrigues Alves de Santana \\ Carla Rodrigues Zanin \\ José Victor Maniglia
}

Faculdade de Medicina de São José do Rio Preto, São José do Rio Preto-SP, Brasil

Resumo: O estudo avaliou as estratégias de enfrentamento, rede e apoio social de pacientes com câncer de cabeça e pescoço atendidos em um hospital do interior paulista. Os 22 participantes responderam a um Questionário Sociodemográfico, Questionário de Hábitos de Vida, Ficha Clínica, Escala Modos de Enfrentamento de Problemas (EMEP) e Medidas de Rede e Apoio Social. Os resultados indicaram que as estratégias de enfrentamento menos utilizadas foram: focalização na emoção $(M=2,08, \mathrm{DP}=0,63)$ e busca por suporte social $(\mathrm{M}=2,62, \mathrm{DP}=0,80)$, a rede social apresentou-se ampla, mas com grau de intimidade baixo, e a média geral do apoio social foi baixa $(M=71, D P=10)$. As principais correlações encontradas foram: apoio emocional e de informação $(r=0,785, p<0,01)$, estratégias focalização no problema e religiosidade $(r=0,579, p<0,05)$. A análise fatorial identificou interação de determinadas estratégias com tipos específicos de apoio social. Discutem-se implicações para as práticas psicológicas visando facilitar a adaptação ao estressor-saúde e melhorar o suporte social dos pacientes.

Palavras-chave: Neoplasias de cabeça e pescoço. Redes sociais. Apoio social. Enfrentamento.

\section{Cancer patients: coping strategies, social network and social support}

\begin{abstract}
This study assessed coping strategies, social network and support in patients, with head and neck cancer, treated in a hospital in the State of Sao Paulo, Brazil. The 22 participants completed a Social Demographic Questionnaire Inquiring on Life Style, Clinical File, the Escala Modos de Enfrentamento de Problemas (EMEP) (Ways of Coping with Problems Scale) with Measures of Social Network and Support. The least used coping strategies were emotion focused-coping (M: 2,08, SD: 0.63) and search for social support (M: 2.62, SD: 0.80), participants had a large network with low level of intimacy, the general average score for social support was low (M: 71, SD: 10). The main correlations found in the study were emotional support and information $(r=0.785, \mathrm{p}<0.01)$, and problem-focused coping and religion $(r=0.579, \mathrm{p}<0.05)$. Factorial analysis identified interactions between certain coping strategies and social support. Implications for psychological practice aimed to improve adjustment to health stressors and social support are discussed.
\end{abstract}

Keywords: Head and neck neoplasms. Social networks. Social support. Coping behavior.

\section{Pacientes con cáncer: enfrentamiento, red social y apoyo social}

Resumen: El estudio evaluó las estrategias del enfrentamiento, red social, apoyo social de pacientes con cáncer de cabeza y cuello atendidos en un hospital del interior paulista. Los participantes contestaron uno Cuestionario Socio-demográfico y de Costumbres de vida, Ficha Clínica, Escala Modos de Enfrentamento de Problemas (EMEP) (Escala Modos de Enfrentamiento de Problemas) y Medidas de Red y Apoyo Social. Las estrategias menos utilizadas fueran enfrentamiento en la emoción (media: 2,08, DP: 0.63) y la busca por soporte social (media=2,62, $\mathrm{DP}=0,80)$. La red social es numerosa, mas con grado de intimidad bajo, lo apoyo social presentó nota media general baja (media $=71, \mathrm{DP}=10$ ). Las principales correlaciones encontradas fueron: apoyo emocional y de información $(\mathrm{r}=0,785, \mathrm{p}<0,01)$, estrategia focalización en el problema y religiosidad $(\mathrm{r}=0,579, \mathrm{p}<0,05)$. El análisis de los componentes principales identificó la interacción de determinadas estrategias con tipos específicos de apoyo social. Debátanse implicaciones para las prácticas psicológicas para propiciar mejor adaptación al estrésor-salud y adecuado soporte social a los pacientes.

Palabras clave: Neoplasias de cabeza y cuello. Redes sociales. Apoyo social. Enfrentamiento. 


\section{Introdução}

O câncer é uma doença que afeta a vida do paciente, seja no aspecto biológico, psicológico ou social, visto de modo geral como uma enfermidade sinônimo de sofrimento e morte (Barbosa, Santos, Amaral, Gonçalves, \& Bruscato, 2004). No âmbito biológico, o paciente se depara com o diagnóstico de uma doença que tem uma evolução geralmente agressiva, com sintomas debilitantes como dor, perda de peso, presença de nódulos (comuns nos cânceres de cabeça e pescoço) e um tratamento prolongado associado a efeitos colaterais desagradáveis de radioterapia e quimioterapia e/ou mutilações em decorrência de cirurgias invasivas (Venturi, Pamplona, \& Cardoso, 2004).

Compreendendo o contexto biopsicossocial do paciente, admite-se que, frente a esta realidade, ele pode enfrentar grandes dificuldades como: alteração da rotina diária em virtude do tratamento, maior dependência de cuidados de terceiros, mudança de hábitos como tabagismo e etilismo, alteração da imagem corporal, isolamento social, entre outras (Amar, Rapoport, Franzi, Bisordi, \& Lehn, 2002; Costa Neto, Araújo, \& Curado, 2000; Nucci, 2003). Esta situação pode culminar em sofrimento psicológico, evidenciado através de sintomas de depressão, ansiedade, manifestação de pensamentos de desesperança, sentimentos de medo e incerteza quanto ao futuro e insatisfação com a imagem corporal (Abby \& Wasserman, 1992, citados por Nucci, 2003; Costa Neto e cols., 2000; Garssen \& Goodkin, 1999, citados por Amar e cols., 2002; Zanin, Corrêa, \& Maniglia, 1999).

De acordo com a condição que o paciente com câncer vive, ele utilizará as estratégias de enfrentamento, entendidas como as habilidades para domínio e adaptação a situações de estresse (Savoia, 1999). O modelo transacional de Lazarus e Folkman (Folkman, Lazarus, Gruen, \& De Longis, 1986) defende que enfrentamento ou coping é o processo no qual o sujeito administra a relação pessoa/ambiente em situações que são percebidas como estressantes. Neste contexto, é feita uma avaliação da realidade para o organismo se preparar adequadamente e responder ao estressor, amenizando-o ou solucionando-o.

O coping pode ser correlacionado com estudos sobre cognições, comportamentos, emoções, reações fisiológicas e situações sociais (Justice, 1988). Quanto a este último item, o ambiente social também é investigado como fator que exerce papel na prevenção contra doenças e na manutenção da saúde (Valla, 1999). A forma como o grupo social percebe o surgimento de uma doença e a evolução do tratamento do câncer influenciam a escolha dos recursos de enfrentamento que o paciente utilizará (Nucci, 2003).

Quando se fala em grupo social, é importante lembrar que os construtos rede social e apoio social são diferentes, embora relacionados entre si. Rede social é o grupo de pessoas no qual o indivíduo tem algum vínculo (Bowling, 1997 citado por Griep, 2003), incluindo os relacionamentos mais próximos (família e amigos íntimos) e relacionamentos formais (outros grupos) (Seeman, 1996). Este conceito compreende: número de pessoas com quem se mantém contato social (parentes, amigos íntimos); a frequiência dessas interações; a condição de ter ou não um(a) companheiro(a); a composição da família e a participação de atividades sociais em grupo (associação, religião, voluntariado) de acordo com Berkman (2000, citado por Griep, Chor, Faerstein, \& Lopes, 2003). Observa-se ainda, que a pessoa pode ter uma rede social e não receber necessariamente apoio desta rede. Na verdade, "a rede social pode ser concebida como a estrutura social através da qual o apoio é fornecido" (Griep, 2003, p. 14).

Por outro lado, o apoio social diz respeito ao aspecto funcional ou qualitativo da rede social (Antunes \& Fontaine, 2005), quer dizer, refere-se a ter alguém com quem contar para receber, por exemplo, auxílio material, emocional ou afetivo, percebendo-se valorizado no grupo de que faz parte segundo Berkman (1984, citado por Griep, 2003). Valla (1999) reafirma esta idéia, definindo apoio social como o fornecimento de informações, auxílio material por grupos e/ou pessoas que estabelecem contatos sistemáticos com um indivíduo, resultando em efeitos 
Santana, J. J. R. A., Zanin, C. R. \& Maniglia, J. V. (2008). Câncer: enfrentamento e apoio social 373

emocionais e comportamentais positivos, sendo, portanto, uma troca na qual ambas as partes são beneficiadas.

Os pesquisadores (Leeuw e cols., 2000) estudaram a associação das variáveis: estratégias de enfrentamento, apoio social e sintomas depressivos em pacientes com câncer de cabeça e pescoço. Eles verificaram que o suporte emocional ineficaz, a rede social insuficiente e o enfrentamento voltado para evitação do problema são preditores de sintomas depressivos durante o tratamento da neoplasia. Desta forma, este estudo teve como finalidade avaliar as estratégias de enfrentamento, a rede social e o apoio social de pacientes diagnosticados com câncer de cabeça e pescoço que são atendidos no ambulatório de um hospital do interior paulista.

\section{Método}

Esta pesquisa foi autorizada pelo Comitê de Ética em Pesquisa da Faculdade de Medicina de São José do Rio Preto-SP, estando, portanto, de acordo com a Resolução CNS 196/96.

Participaram deste estudo pacientes atendidos no Ambulatório de Otorrinolaringologia do Hospital de Base de São José do Rio Preto (cadastrados na Secretaria do Estado da Saúde - Registro Hospitalar de Câncer - Fundação Oncocentro de São Paulo), que receberam o diagnóstico de câncer de cabeça e pescoço, de acordo com a $3^{\mathrm{a}}$ edição da Classificação Internacional de Doenças para Oncologia (Organização Mundial da Saúde, 2005), no período de janeiro de 2005 a junho de 2006.

Foram incluídos pacientes de ambos os sexos, sem restrição de idade, escolaridade, nível sócio-econômico, raça, religião e estado civil. Os critérios de exclusão foram: (1) recusa em participar da pesquisa; (2) comprometimento neurológico ou psiquiátrico; (3) indivíduo apresentando alterações de consciência (coma) ou comprometimento físico (paciente entubado, acamado ou com deficiência auditiva nãocorrigida) que impossibilitasse ser entrevistado.

Os instrumentos utilizados foram:

(1) Questionários Sociodemográfico e de Hábitos de Vida (idade, sexo, estado civil, escolari- dade, situação ocupacional, tipo de moradia, hábitos tabagista e etilista);

(2) Ficha de dados clínicos (localização anatômica do câncer e estadiamento);

(3) Escala Modos de Enfrentamento de Problemas: adaptada para o português por Gimenes e Queiroz (1997), análise fatorial feita por Seidl, Tróccoli e Zannon (2001). Escala de 45 itens, respondidos segundo uma escala Likert de cinco pontos, agrupados nos fatores: enfrentamento focalizado no problema (18 itens: comportamentos para controlar o problema causador do estresse); enfrentamento focalizado na emoção (15 itens: tentativas de adequar a resposta emocional ao problema); busca de suporte social (cinco itens: procura por apoio instrumental, emocional ou de informação) e busca de práticas religiosas/pensamento fantasioso (sete itens: comportamentos religiosos, pensamentos de esperança que servem para auxiliar no enfrentamento do problema);

(4) Medidas de Rede e Apoio Social: validadas por Griep (2003). O bloco de rede social possui os seguintes itens: quantidade de parentes e amigos íntimos; situação conjugal; condição de morar sozinho ou não; participação em atividades em grupo (esporte, reuniões de pessoas ou atividade voluntária). O questionário de apoio social é constituído de 19 questões (com respostas em uma escala de cinco pontos) abrangendo cinco dimensões: apoio material (quatro perguntas: provisão de recursos práticos e ajuda material); afetivo (três perguntas: demonstrações físicas de amor e afeto); emocional (quatro perguntas: expressões de afeto positivo, compreensão e sentimentos de confiança); interação social positiva (quatro perguntas: disponibilidade de pessoas para divertir-se ou relaxar) e informação (quatro perguntas: disponibilidade de pessoas para a obtenção de conselhos ou orientações).

Através do cadastro oficial de pacientes do hospital elaborou-se uma lista de pessoas que tinham os critérios de inclusão no estudo. Para explicar os objetivos da pesquisa e marcar data para coleta de dados após a adesão, os pacientes foram contatados pelos seguintes meios: pessoalmente, por telefone ou 
carta, necessariamente nesta ordem, caso a tentativa anterior de contato tenha falhado. Este procedimento foi adotado para agilizar a comunicação dos pesquisadores com os pacientes, privilegiando o contato pessoal e, assim, sanar as dúvidas sobre o estudo de forma mais eficaz. Após explicações sobre o objetivo do estudo e obtenção do consentimento para ingressar na pesquisa, em duas vias, os participantes responderam aos instrumentos. Garantiu-se uma cópia do Termo de Consentimento Livre e Esclarecido, assinado tanto pelo participante quanto pelo pesquisador, a cada ingressante na pesquisa.

Cumpriram os critérios de inclusão do estudo 111 pacientes cadastrados no Registro Hospitalar do Câncer com os critérios diagnósticos já descritos. Destes, foram excluídos: 26 pacientes cujo óbito não fora registrado no cadastro oficial; 24 não compareceram no horário marcado para a coleta de dados ou se recusaram a participar do estudo; 14 constavam com endereço e/ou telefone incorreto no banco de dados hospitalar; 14 apresentavam algum tipo de comprometimento neurológico, cognitivo ou psiquiátrico que impossibilitou compreensão adequada das perguntas do protocolo da pesquisa; cinco apresentavam deficiência auditiva não-corrigida; quatro pessoas encontravam-se limitadas fisicamente inviabilizando participação no estudo (acamadas ou internadas no hospital); um encontrava-se na condição de presidiário no momento do estudo e outro foi excluído do Registro Hospitalar do Câncer após exames que refutaram o diagnóstico da doença. Desta forma, a amostra foi composta por 22 pacientes.

Os dados foram analisados qualitativa e quantitativamente, sendo adotado nível de significância $\mathrm{p} \leq 0,05$, apresentados em estimativa de percentual (intervalo de confiança $95 \%$ ) e foram confrontados com $50 \%$ por meio do teste exato para uma proporção. As variáveis "apoio social" e "estratégias de enfrentamento" foram analisadas por correlação e por Análise dos Componentes Principais.

\section{Resultados e Discussão}

Dos 22 participantes, 19 (86\%) eram do sexo masculino e três (14\%) feminino. A média de idade foi 57 anos $(\mathrm{DP}=13)$. Quanto ao estado civil, 13 entrevistados $(59 \%)$ declararam viver em união estável. A amostra apresentou escolaridade baixa, ou seja, nove pessoas (41\%) entre um e quatro anos de estudos. Referente às atividades laborais, 17 (77\%) disseram estar afastados ou aposentados da função de trabalho. Onze pessoas (58\%) responderam residir em casa própria.

Quanto ao hábito tabagista, 17 (77\%) são extabagistas, três (14\%) declararam ser tabagistas e dois $(9 \%)$ nunca fumaram. A média de persistência do hábito tabagista foi 37 anos $(\mathrm{DP}=15)$. Doze pacientes $(57 \%)$ são ex-etilistas, seis $(29 \%)$ são etilistas e quatro (18\%) não desenvolveram o hábito de ingerir bebidas alcoólicas. A média de duração do hábito etilista é de 27 anos $(\mathrm{DP}=15)$. Os resultados deste trabalho evidenciam a influência de hábitos de vida (tabagismo e etilismo) no desenvolvimento do câncer, relação bem estabelecida pela literatura (Sartor e cols., 2007).

Este resultado tem valor preditivo ruim, principalmente para os que mantêm o hábito tabagista, pois existem evidências da associação negativa entre este comportamento e a qualidade de vida para os pacientes com câncer de cabeça e pescoço (Duffy e cols., 2007). Há necessidade urgente de intervenção programada voltada para a cessação deste hábito, como propõe o Ministério da Saúde em seu Programa Nacional de Controle do Tabagismo (Brasil, 2003).

Quanto aos dados clínicos da amostra estudada, foi estimado que quatro pacientes $(18,18 \%)$ foram diagnosticados no estadiamento TI do câncer, seis $(27,27 \%)$ no estadiamento TII, outros seis $(27,27 \%)$ no estadiamento TIII, cinco $(22,73 \%)$ no estadiamento TIV e um paciente $(4,55 \%)$ com estadiamento não definido até o momento da coleta de dados (TX).

Comparando os escores médios das estratégias de enfrentamento do estressor saúde da amostra estudada e da população geral (da amostra de validação do instrumento), verificam-se os resultados apresentados na Figura 1. 
Santana, J. J. R. A., Zanin, C. R. \& Maniglia, J. V. (2008). Câncer: enfrentamento e apoio social 375

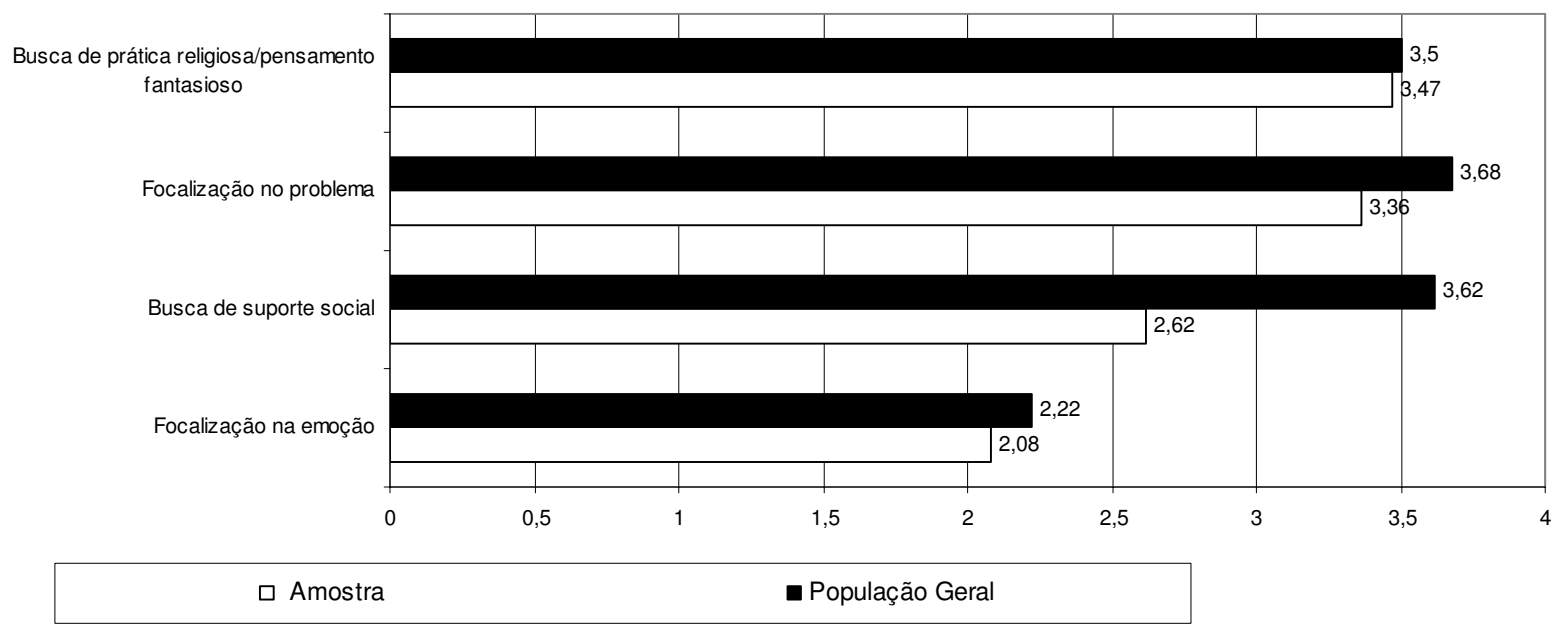

Figura 1. Médias dos fatores da Escala Modos de Enfrentamento de Problemas da amostra estudada e da população geral (de validação da escala).

Observa-se que na amostra estudada os escores médios das estratégias de enfrentamento do estressor-saúde figuram dentro do esperado, se comparados aos dados da população geral, exceto quanto à estratégia de enfrentamento "busca de suporte social". O escore médio da amostra para esta estratégia $(\mathrm{M}=2,62 ; \mathrm{DP}=0,80)$ foi significativamente menor que o escore médio da população geral $(\mathrm{M}=3,62 ; \mathrm{DP}=0,67)$.

A estratégia de enfrentamento "busca de suporte social" é de extrema importância, principalmente quanto à busca por informações sobre o diagnóstico e o tratamento da doença. Van der Molen (1999), estudou pacientes com câncer e revelou que as informações sobre o tratamento e sobre recursos assistenciais oferecidos pela comunidade para auxiliálo podem reduzir a tensão e ansiedade advindas da vivência do câncer.

Considerando que boa parte da amostra é dependente do álcool ou é ex-etilista, levanta-se a hipótese de que estes pacientes recorrem menos ao suporte social para enfrentamento da doença em virtude do padrão comportamental geralmente desempenhado por pacientes etilistas, de extrema independência, autonomia e agressividade, elementos que se contrapõem ao comportamento de solicitar ajuda. Além disso, o etilismo traz consequiências para o ambiente familiar, como geração de conflito e agressões, situações que impedem o fornecimento adequado de suporte social (Figlie, Bordin, \& Laranjeira, 2004; Hannickel, Zago, Barbeira, \& Sawada, 2002).
Dessa forma, o paciente pode ter rede e apoio social disponíveis, mas não possuir habilidades para recorrer a este ambiente social ou então, pelo impacto familiar do etilismo não possuir contexto social que lhe forneça suporte frente aos estressores. Como afirmaram Figlie e cols. (2004, p. 44), "é difícil determinar o que é causa e o que é consequiência". Infelizmente, a abrangência destas suposições inviabiliza a testagem neste estudo restrito. Novos trabalhos com esta temática contribuirão para maiores esclarecimentos.

Outra hipótese aborda o fato de que o pouco uso da estratégia suporte social ocorre porque os pacientes se afastam das pessoas devido à aparência física alterada pelo tratamento do câncer (mutilações cirúrgicas, emagrecimento), como verificaram resultados de estudos sobre pacientes laringectomizados (Barbosa e cols., 2004; Hannickel e cols., 2002; Zago, Sawada, Stopa, \& Martinez, 1998). Novamente, estas variáveis que aqui não foram abordadas merecem atenção em estudos posteriores.

Nota-se que tanto para a amostra do presente estudo quanto para a população de validação da escala, a estratégia de enfrentamento com menor escore foi a focalização na emoção. Supõe-se que este dado é esperado quando se considera que estes pacientes apresentam comportamentos de busca de cuidado com a saúde (focalização no problema), já que foram contatados no serviço hospitalar. Entretanto, levanta-se um questionamento sobre o impacto do 
pouco uso desta estratégia para ambos os contextos. Uma vez que o câncer de cabeça e pescoço envolve a experiência de situações altamente estressoras que um paciente com outra doença crônica não vivencia, o impacto da falta de focalização na emoção prejudicaria mais um grupo que outro?

Entre as situações estressoras que um paciente com câncer de cabeça e pescoço vivencia, cita-se como um exemplo a expectativa quanto ao prognóstico. Estimativas revelam que a taxa de sobrevida do câncer de cavidade oral em dois anos é de $57 \%$ no estágio III e 45\% no estágio IV (Jotz e cols., 2007). Este quadro se agrava quando reportamos a expectativa de sobrevida de pacientes após recidiva intratável de câncer de cabeça e pescoço, que apresenta mediana de 17 semanas (Amar, Ortellado, Franzi, Curioni, \& Rapoport, 2005).

Assim, na amostra do presente estudo seis pacientes $(27,27 \%)$ foram diagnosticados no estadiamento III e cinco $(22,73 \%)$ no estadiamento $\mathrm{IV}$, e que, por isso, necessitam adequar a resposta emocional ao problema, pois a situação possivelmente será avaliada da forma pela qual pouco pode ser feito para modificar as condições de dano e terminalidade. Portanto, talvez o maior uso desta estratégia favoreceria melhor adaptação ao problema, situando este recurso como um fator de proteção a transtornos psiquiátricos decorrentes do estresse vivenciado. A estratégia de enfrentamento voltada para a regulação da emoção é adequada para gerir a condição de ansiedade que o paciente pode vivenciar frente aos tratamentos, como a cirurgia, radioterapia ou quimioterapia. Os pesquisadores (Medeiros \& Peniche, 2006) já apresentaram evidências da influência do enfrentamento baseado na emoção para controle da ansiedade em pacientes cirúrgicos.

Uma intervenção direcionada para o desenvolvimento de estratégias de focalização na emoção pode ser altamente benéfica e acrescentar conhecimentos ao pouco que se sabe sobre os cuidados paliativos com pacientes com câncer de cabeça e pescoço (principalmente quanto aos aspectos psicossociais), aperfeiçoando as práticas executadas por centros especializados de atendimento (Ledeboer, Van Der Velden, De Bôer, Feenstra, \& Pruyn, 2006).

Os pesquisadores Ibarra, Suárez e Forero (2004) reportaram uma experiência de intervenção com pacientes com câncer. A atuação psicológica foi pautada nos conhecimentos da terapia cognitivocomportamental, que subsidiou várias técnicas utilizadas no programa, dentre elas, o treino de habilidades de enfrentamento à condição de prognóstico ruim da doença. Como resultados obtidos, destacam-se a expressão de sentimentos, a reflexão sobre o sentido da vida, melhora na habilidade de comunicação (principalmente com familiares) e melhor capacidade para tomada de decisões.

Um estudo recente (He \& Liu, 2005) demonstrou que a adequação da resposta emocional ao problema de saúde está positivamente correlacionada com uma boa qualidade de vida do paciente com câncer localizado na nasofaringe. Neste ponto, é conveniente a sugestão de estudos, que avaliem intervenções psicológicas voltadas para o desenvolvimento desta estratégia de enfrentamento, nestes pacientes.

Acerca da medida de rede social, verificou-se que, em média, a quantidade de parentes íntimos referida foi nove $(\mathrm{DP}=14)$ e de amigos íntimos, seis $(\mathrm{DP}=12)$. Treze pessoas $(59 \%)$ declararam viver situação conjugal do tipo união estável. A quantidade média de moradores das residências do paciente foi três $(\mathrm{DP}=1)$, sendo que três pacientes $(14 \%)$ moram sozinhos. Foi estimado que 16 pessoas (73\%) não participaram de nenhuma atividade esportiva no último ano; 17 (77\%) não se reuniram em associação de pessoas neste período e 18 (82\%) não participaram de atividade voluntária.

Os resultados sobre a rede social foram satisfatórios para os 22 participantes da pesquisa, no que se refere ao número de pessoas com as quais os pacientes têm contato social. Entretanto, a fragilidade destas interações quanto ao grau de intimidade e reciprocidade é preocupante, pois os pacientes participam menos freqüentemente de situações em grupo e, portanto, têm menos contato com as pessoas que fazem parte de seu ambiente social. Meneses e Sarriera (2005), em uma revisão teórica, reuniram evidências sobre a influência positiva que a rede social tem para a adesão e êxito no tratamento, principalmente quanto aos aspectos de auto-cuidado e ajustamento emocional à enfermidade. Holloway e cols. (2005) também destacaram a importância da rede social para a melhoria da qualidade de vida de pacientes com câncer de cabeça e pescoço. Provavelmente o resultado da presente pesquisa se deve à interferência de variáveis como limitações físicas 
Santana, J. J. R. A., Zanin, C. R. \& Maniglia, J. V. (2008). Câncer: enfrentamento e apoio social 377

provocadas pela doença, vivência do estigma do câncer, entre outras, não avaliadas nesta pesquisa.

Sobre a variável apoio social, foi estimado que a média obtida dos pacientes para a dimensão apoio afetivo foi $85(\mathrm{DP}=18)$; apoio material com escore médio de $80(\mathrm{DP}=22)$; o escore médio para apoio emocional foi 69 ( $\mathrm{DP}=20)$; do apoio de informação $65(\mathrm{DP}=21)$ e, por fim, escore médio do apoio social do tipo "interação social positiva" foi $59(\mathrm{DP}=21)$. A média geral para a variável apoio social foi 71 $(\mathrm{DP}=10)$. Todos estes escores estão abaixo dos observados no estudo de validação da escala (Griep, 2003), confirmando que os pacientes avaliados não estão totalmente satisfeitos com o apoio recebido pelo seu contexto social.

Pesquisando uma amostra de pacientes que também sofrem neoplasia de cabeça e pescoço, Derks, Lieuw, Winnubst e Horkijk (2004) demonstraram que o apoio social percebido diminui significativamente como consequiência do tratamento da neoplasia, afe- tando, igualmente, pacientes idosos e jovens. Pesquisas futuras no contexto deste trabalho, podem ser úteis, para investigar o apoio social antes e após um determinado período de tratamento da doença. Outro estudo, também realizado com pacientes com câncer de cabeça e pescoço, mostrou evidências da relação entre apoio social satisfatório e qualidade de vida (Llewellyn, Mcgurk, \& Weinman, 2005). Dados empíricos apontam correlação positiva entre altos escores do apoio social percebido com variáveis como capacidade de expressão da fala; avaliação positiva da aparência física; baixos escores de sintomas depressivos e boa saúde mental. Os pesquisadores concluem que intervenção voltada para a melhoria da percepção do suporte social recebido pode aumentar a sobrevivência destes pacientes (Karnell, Christensen, Rosenthal, Magnuson, \& Funk, 2006).

As correlações entre as variáveis apoio social e estratégias de enfrentamento estão apresentadas na Tabela 1.

Tabela 1

Coeficientes de correlação de Pearson entre o apoio social percebido e as estratégias de enfrentamento da amostra

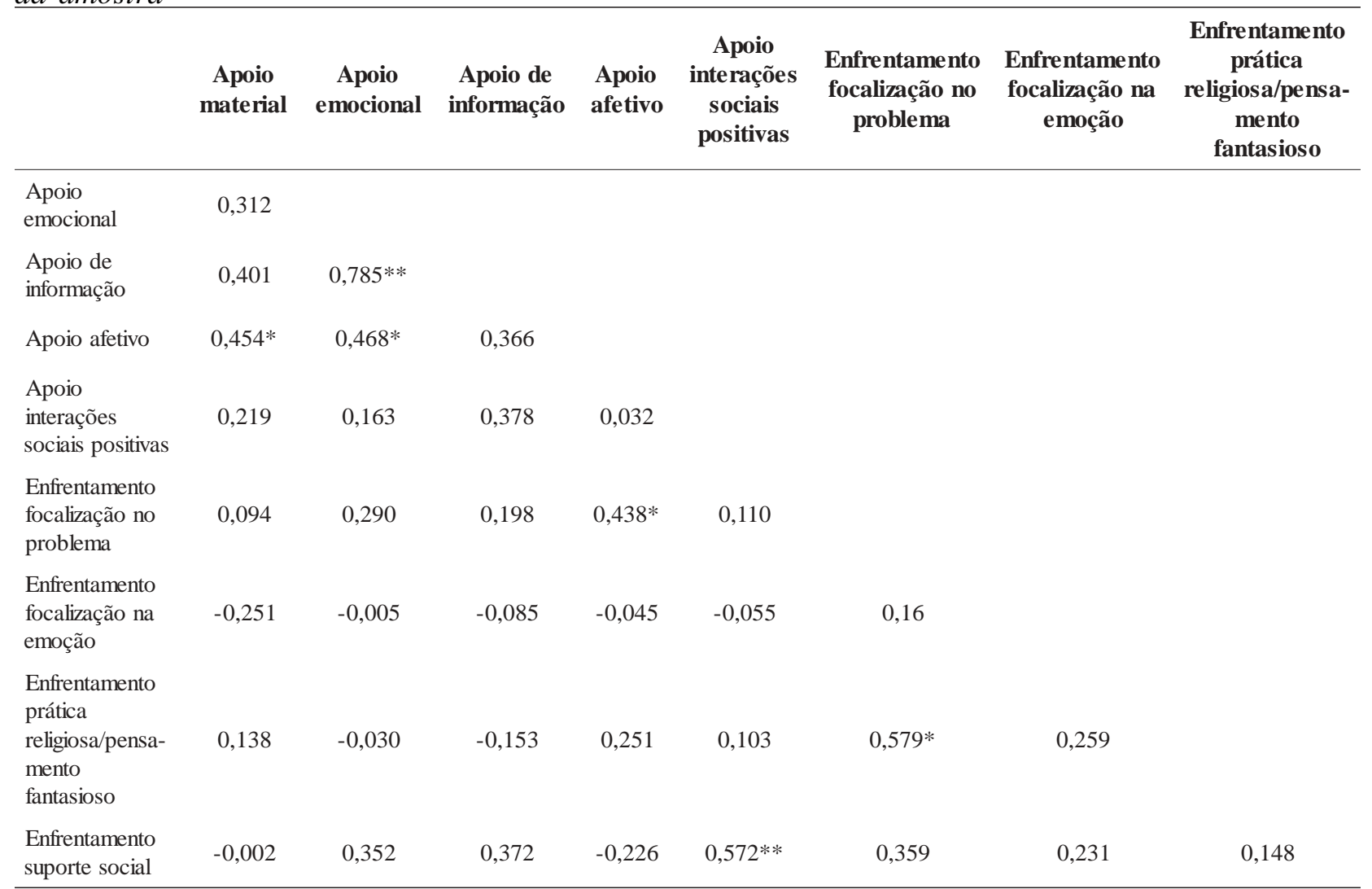


As associações estatisticamente significativas (aquelas cujo valor-p é superior a 0,01 ou 0,05 ) serão discutidas a seguir. Notam-se correlações positivas significativas entre apoio emocional e apoio de informação $(r=0,785 ; p<0,01)$ e também afetivo $(r=0,468$; $\mathrm{p}<0,05)$. Assim, as pessoas com maiores escores quanto à percepção de apoio de informação e afetivo, obtiveram escores igualmente elevados para apoio emocional. Este resultado é considerado positivo, pois evidencia que o ambiente social de alguns pacientes é percebido por eles como acolhedor, onde existem demonstrações físicas de afeto e disponibilidade de aconselhamentos e sugestões para lidar com as dificuldades, além de ser próprio para a expressão de sentimentos de confiança, empatia e carinho. Podese considerar que este apoio social satisfatório exerce função mediadora do processo saúde-doença, minimizando o impacto que o câncer exerce na vida do paciente, estando associado também à saúde mental. Outras evidências empíricas apontam que tanto o apoio emocional quanto o de informação são igualmente eficazes na adaptação ao câncer (Helgeson $\&$ Cohen, 1996). Pesquisas futuras podem fornecer conhecimentos sobre a relação entre o apoio social e o prognóstico da doença (Marmot \& Wilkinson, 2006).

Foram encontradas, também, correlações positivas entre apoio afetivo e material $(r=0,454 ; p<0,05)$ e a estratégia de enfrentamento focalização no problema ( $\mathrm{r}=0,438 ; \mathrm{p}<0,05)$, indicando que as pessoas cujo comportamento é focalizado no problema também caracterizam o seu grupo social enquanto fornecedor de apoio material e afetivo. É importante lembrar que os comportamentos direcionados ao problema objetivando a busca de saúde (adesão ao tratamento, cessação do tabagismo e etilismo) podem não obter resultados positivos se o grupo social não valorizar estas mudanças, oferecendo o reforçamento ou pu- nição necessários para desenvolvimento de comportamentos de valorização da saúde (Bandura, Azzi, \& Polydoro, 2008; Berkman \& Glass, 2000 citados por Griep, 2003; Rosas \& Baptista, 2002; Skinner, 2000).

A variável apoio social do tipo interações sociais positivas foi significativamente associada de forma positiva com a estratégia de enfrentamento busca por suporte social $(r=0,572 ; \mathrm{p}<0,01)$. Isto quer dizer que, na vivência do estressor-saúde, os pacientes que lançam mão da estratégia busca de suporte social tendem a contar com pessoas com quem possam relaxar e se divertir. $\mathrm{O}$ acesso a este tipo de vínculo social pode proteger os pacientes de sentimentos negativos advindos das perdas inerentes à doença, favorecendo o bem-estar e a saúde mental destas pessoas (Guedea e cols., 2006).

Em conformidade com os dados da literatura (Seidl e cols., 2001) foi verificada correlação positiva estatisticamente significativa entre estratégia focalização no problema com prática religiosa/pensamento fantasioso $(r=0,579 ; \mathrm{p}<0,05)$; apontando para a consideração de que estas são estratégias que exercem funções complementares no enfrentamento do estresse para esta amostra.

A fim de analisar as inter-relações entre as variáveis "apoio social” e "estratégias de enfrentamento", de modo que elas sejam representadas por um conjunto de dimensões, foi realizada a extração de fatores através do método de análise dos componentes principais. Para isto, foram utilizados os seguintes critérios: (1) número de componentes com autovalor (eigenvalue) maior que 1,5; (2) cada fator explicando no mínimo $10 \%$ da variância total; (3) apenas itens com carga fatorial superior a 0,40 (positiva ou negativa) foram incluídos nos fatores. Os dados estão apresentados na Tabela 2. 
Santana, J. J. R. A., Zanin, C. R. \& Maniglia, J. V. (2008). Câncer: enfrentamento e apoio social 379

Tabela 2

Análise dos componentes principais do apoio social percebido e estratégias de enfrentamento da amostra

\begin{tabular}{lccc}
\hline Eigenvalue & 2,90 & 1,76 & 1,60 \\
\% variância & 32,21 & 19,53 & 17,80 \\
\% acumulada & 32,21 & 51,74 & 69,52 \\
\hline \multicolumn{1}{c}{ Variáveis } & \multicolumn{3}{c}{ Componentes } \\
& $\mathbf{1}$ & $\mathbf{2}$ & $\mathbf{3}$ \\
\hline Apoio material & 0,550 & $-0,393$ & 0,273 \\
Apoio emocional & 0,795 & $-0,243$ & $-0,0071$ \\
$\begin{array}{l}\text { Apoio de informação } \\
\text { Apoio afetivo }\end{array}$ & 0,801 & $-0,355$ & $-0,253$ \\
$\begin{array}{l}\text { Apoio interações sociais } \\
\text { positivas }\end{array}$ & 0,602 & $-0,181$ & 0,652 \\
$\begin{array}{l}\text { Estratégia focalização no } \\
\text { problema }\end{array}$ & 0,507 & 0,101 & $-0,529$ \\
$\begin{array}{l}\text { Estratégia focalização na } \\
\text { emoção }\end{array}$ & 0,589 & 0,541 & 0,333 \\
$\begin{array}{l}\text { Estratégia prática } \\
\text { religiosa/pensamento } \\
\text { fantasioso }\end{array}$ & 0,292 & 0,684 & 0,452 \\
$\begin{array}{l}\text { Estratégia busca de } \\
\text { suporte social }\end{array}$ & 0,521 & 0,433 & $-0,0048$ \\
\hline
\end{tabular}

Método de extração dos fatores: análise dos componentes principais

Esta análise mostrou que, com $32 \%$ da variância total e eigenvalue 2,90, o primeiro fator (que mais contribui para o total da variância explicada) pondera para uma "Tendência Global", ou seja, não há contraposição entre os itens. Este dado revela que os pacientes utilizam formas diversificadas de apoio social e estratégias de enfrentamento da condição de enfermidade, sendo que esta maleabilidade tem aspecto positivo, uma vez que, se um dado recurso não alcançar o objetivo pretendido, outro será mobilizado com este fim. O paciente que possui relações diversificadas para receber ajuda emocional, cognitiva e material para enfrentar situações difíceis (Caplan, 1974 citado por Griep, 2003) e recorre a vários comportamentos com funções voltadas para a adaptação ao contexto, obterá maior êxito no gerenciamento, controle ou redução do evento estressor (Trentini, Silva, Valle, \& Hammerschmidt, 2005) e terá maior senso de auto-eficácia para lidar com estas situações (Bandura e cols., 2008; Seidl, Melchíades, Farias, \& Brito, 2007).

No segundo fator, com $20 \%$ da variação total e eigenvalue 1,76, existe uma tendência de um grupo para utilizar várias estratégias de enfrentamento dos problemas mais do que perceber o ambiente social como fornecedor de apoio. Chamaremos este fator de "Multiplicidade de Estratégias de Enfrentamento versus Suporte Social Insatisfatório". Este resultado reafirma os dados anteriormente descritos, demonstrando que a rede social de alguns pacientes é ineficaz para subsidiá-los de apoio e suporte necessários na situação de adoecimento. Evidências empíricas demonstraram que a inclusão num contexto de apoio social (emocional e prático) e também participação em atividades sociais de lazer e passatempos, são itens promotores de saúde em pacientes com câncer de cabeça e pescoço (Björklund, Sarvimäki, \& Berg, 2008). Ações multidisciplinares da equipe de saúde voltadas para este fim são sugestões que favoreceriam melhor qualidade de vida destes pacientes (Hassanein, Musgrove, \& Bradbury, 2001).

Com $18 \%$ da variância total e eigenvalue 1,60, o terceiro fator dispõe a estratégia de enfrentamento busca de suporte social e o apoio de interações sociais positivas em contraposição ao apoio afetivo e prática religiosa/pensamento fantasioso. Esta polarização revela que um grupo de pacientes que busca o suporte social para enfrentamento dos problemas e participa de relações que envolvem situações prazerosas e de lazer não percebe receber apoio afetivo e busca menos práticas religiosas. Embora não foram encontradas justificativas teóricas consistentes para este fator, levanta-se a hipótese de que determinados tipos de estratégias de enfrentamento estão interligados a dimensões específicas do apoio social. Isto pode ser resultado das mudanças nas necessidades do paciente ao longo do tratamento (estadiamento do câncer; efeitos adversos da radioterapia e quimioterapia; duração do tratamento; entre outras). Mccoll, Lei e Skinner (1995) estudaram a relação entre apoio social e coping de pacientes com lesão medular ao longo de 
um ano. Os pesquisadores verificaram que as estratégias de enfrentamento não variaram com o tempo, mas o suporte social modificou ao longo deste período, variando de apoio de informação no começo do tratamento para apoio emocional no fim dos doze meses. Os autores afirmam que estes pacientes devem aperfeiçoar suas estratégias de enfrentamento no período de adaptação para lidar com as demandas de longo prazo como alteração da estrutura de apoio social e com as necessidades cotidianas (de locomoção, cuidados pessoais).

Um estudo com pacientes com câncer de cabeça e pescoço indicou que as estratégias de enfrentamento da doença variam conforme o momento do tratamento (Vidhubala, Latha, Ravikannan, Mani, $\&$ Karthikesh, 2005). A avaliação das estratégias de enfrentamento ao longo do tempo é uma medida já defendida por Lazarus (1993 citado por Ribeiro \& Rodrigues, 2004) quando fundamentou o construto de coping. Para ele, a perspectiva do enfrentamento é orientada para o contexto e para o processo, ou seja, é preciso identificar as estratégias utilizadas pelo indivíduo em vários contextos e momentos com a finalidade de verificar a estabilidade e as alterações das cognições e comportamentos com relação ao problema ao longo do tempo.

Assim, estudos semelhantes com pacientes com câncer de cabeça e pescoço são relevantes para avaliar a interação entre as estratégias de enfrentamento e o apoio social, bem como a variação destes aspectos ao longo do tratamento e propor medidas de intervenção com estes pacientes visando a melhoria da qualidade de vida.

\section{Considerações finais}

O presente estudo alcançou os objetivos almejados. Ele identificou que a estratégia de enfrentamento dos pacientes avaliados considerada insuficiente foi a busca por suporte social, situação paralela ao fato de os participantes avaliarem como insatisfatórias a rede social e o apoio social.

Importantes associações entre as variáveis foram verificadas, o que permitiu levantar demandas para o trabalho do psicólogo neste contexto. Frente a isto, algumas possibilidades de intervenção psicológica junto a estes pacientes para auxílio no enfrentamento do câncer de cabeça e pescoço foram abordadas. Para os pacientes que persistem no hábito tabagista, por exemplo, é evidente a necessidade de um trabalho sistematizado, talvez nos moldes do Programa Nacional de Controle do Tabagismo, proposto pelo Ministério da Saúde (Brasil, 2003). Outra necessidade levantada foi desenvolver estratégias de enfrentamento ao câncer mais diversificadas e eficazes. A literatura traz importantes sugestões neste sentido, como a terapia cognitivo-comportamental no treino de habilidades de enfrentamento (Ibarra e cols., 2004); a realização de grupos de apoio e informativos (Van der Molen, 1999; Helgeson \& Cohen, 1996); intervenções voltadas para a imagem corporal alterada (Hannickel e cols., 2002); para a expressão e regulação das emoções (Medeiros \& Peniche, 2006; Ledeboer e cols., 2006); intervenção direcionada para familiares e pessoas próximas ao paciente (Bandura e cols., 2008), entre outras. Verificouse, também, a necessidade de realização de um trabalho multidisciplinar para fortalecer a rede social dos pacientes e melhorar a percepção de apoio social recebido. Assim, o paciente terá maior oportunidade de falar sobre os problemas advindos da doença (Östberg \& Lennartsson, 2007) e também, participar de atividades sociais de lazer que comprovadamente são itens promotores de saúde em pacientes com câncer de cabeça e pescoço (Björklund e cols., 2008).

Através da presente pesquisa, novos temas de investigação foram levantados, como por exemplo: verificar a relação entre a estratégia busca de suporte social e o comportamento etilista; avaliar o impacto psicológico da aparência física alterada pela neoplasia; identificar os efeitos de intervenções psicológicas no tratamento da doença e comparar escores das variáveis apoio social e estratégias de enfrentamento ao longo do tempo.

A amostra restrita foi uma limitação deste estudo. As dificuldades de acesso aos participantes foram identificadas, o que contribui para que, em estudos posteriores elas sejam contornadas. A presente pesquisa contribuiu efetivamente para o avanço do conhecimento em Psicologia da Saúde, pois evidenciou a importância da investigação de variáveis como enfrentamento, rede e apoio social de pessoas com câncer, especialmente para as que foram aqui avalia- 
Santana, J. J. R. A., Zanin, C. R. \& Maniglia, J. V. (2008). Câncer: enfrentamento e apoio social 381

das. Com os dados levantados, o psicólogo pode planejar práticas voltadas para a melhoria da qualidade de vida destes pacientes que sofrem neoplasias de cabeça e pescoço.

\section{Referências}

Amar, A., Ortellado, D. K., Franzi, S. A., Curioni, O. A., \& Rapoport, A. (2005). Sobrevida após recidiva intratável do carcinoma epidermóide de cabeça e pescoço. Revista do Colégio Brasileiro de Cirurgiões, 32, 267-269.

Amar, A., Rapoport, A., Franzi, S. A., Bisordi, C., \& Lehn, C. N. (2002). Qualidade de vida e prognóstico nos carcinomas epidermóides de cabeça e pescoço. Revista Brasileira de Otorrinolaringologia, 68, 400-403.

Antunes, C., \& Fontaine, A. M. (2005). Percepção de apoio social na adolescência: Análise fatorial confirmatória da Escala Social Support Appraisals. Paidéia (Ribeirão Preto), 15(32), 355-366.

Bandura, A., Azzi, R. G., \& Polydoro, A. (Orgs.). (2008). Teoria social cognitiva: Conceitos básicos. Porto Alegre: Artmed.

Barbosa, L. N. F., Santos, D. A., Amaral, M. X., Gonçalves, A. J., \& Bruscato, W. L. (2004). Repercussões psicossociais em pacientes submetidos a laringectomia total por câncer de laringe: Um estudo clínico-qualitativo. Revista da Sociedade Brasileira de Psicologia Hospitalar, 7(1), 45-58.

Björklund, M., Sarvimäki, A., \& Berg, A. (2008). Health promotion and empowerment from the perspective of individuals living with head and neck cancer. European Journal of Oncology Nursing, $12,26-34$.

Brasil. Ministério da Saúde. Instituto Nacional do Câncer. (2003). Programa Nacional de Controle do Tabagismo e outros Fatores de Risco de Câncer: Modelo lógico e avaliação (2a ed.). Rio de Janeiro: INCA.
Costa Neto, S. B., Araújo, T. C. C. F., \& Curado, M. P. (2000). Avaliação da qualidade de vida de pessoas portadoras de câncer de cabeça e pescoço. Acta Oncológica Brasileira, 20, 96-104.

Derks, W., Leeuw, R. D., Winnubst, J., \& Horkijk, G. J. (2004). Elderly patients with head and neck cancer: Physical, social and psychological aspects after one year. Acta Oto-Laryngologica, 124, 509-514.

Duffy, S. A., Ronis, D. L., Valenstein, M., Fowler, K. E., Lambert, M. T., Bishop, C., \& Terrel, J. E. (2007). Depressive symptoms, smoking, krinking, and quality of life among head and neck cancer patients. Psychosomatics, 18, 142-148.

Figlie, N., Bordin, S., \& Laranjeira, R. (2004). Aconselhamento em dependência química (Vol. 1). São Paulo: Roca.

Folkman, S., Lazarus, R. S., Gruen, R. J., \& De Longis, A. (1986). Appraisal, coping, health status and psychological symptons. Journal of Personality and Social Psychology, 50, 571-579.

Gimenes, M. G. G., \& Queiroz, B. (1997). As diferentes fases de enfrentamento durante o primeiro ano após a mastectomia. In M. G. G. Gimenes \& M. H. Fávero (Orgs.), A mulher e o câncer (pp. 173195). Campinas, SP: Editorial Psy.

Griep, R. H. (2003). Confiabilidade e validade de instrumentos de medida de rede social e de apoio social utilizados no Estudo Pró-Saúde. Tese de doutorado não-publicada, Fundação Oswaldo Cruz, Escola Nacional de Saúde Pública, Rio de Janeiro, RJ.

Griep, R. H., Chor, D., Faerstein, E., \& Lopes, C. (2003). Confiabilidade teste-reteste de aspectos da rede social no Estudo Pró-Saúde. Revista de Saúde Pública, 37, 379-385.

Guedea, M. T. D., Albuquerque, F. J. B., Tróccoli, B. T., Noriega, J. A. V., Seabra, M. A. B., \& Guedea, R. L. D. (2006). Relação do bem-estar subjetivo, estratégias de enfrentamento e apoio social em idosos. Psicologia: Reflexão e Crítica, 19, 301-308. 
Hannickel, S., Zago, M. M. F., Barbeira, C. B. S., \& Sawada, N. O. (2002). O comportamento dos laringectomizados frente à imagem corporal. Revista Brasileira de Cancerologia, 48, 333-339.

Hassanein, K. A. A. M., Musgrove, B. T., \& Bradbury, E. (2001). Functional status of patients with oral cancer and its relation to style of coping, social support and psychological status. Britsh Journal of Oral and Maxillofacial Surgery, 39, 340-345.

He, G., \& Liu, S. (2005). Quality of life and coping styles in chinese nasopharyngeal câncer patiens after hospitalization. Cancer Nursing, 28, 179-186.

Helgeson, V. S., \& Cohen, S. (1996). Social support and adjustment to cancer: Reconciling descriptive, correlational, and intervention research. Health Psychology, 15, 135-148.

Holloway, R. L., Hellewell, J. L., Marbella,A. M., Layde, P. M., Myers, K. B., \& Campbell, B. H. (2005). Psychosocial effects in long-term head and neck cancer survivors. Head \& Neck, 27, 281-288.

Ibarra, A. C. R., Suárez, R. Y. R., \& Forero, M. R. (2004). Intervención familiar para el manejo psicológico en pacientes oncológicos con mal prognóstico en el Hospital de La Misericordia. Revista Colombiana de Psicología, 13, 90-101.

Jotz, G. P., Damazzini, R., Deboni, M., Creher, M. V., Demoni, A. L. S., \& Zettler, C. G. (2007). Prognóstico em dois anos do câncer de cavidade oral. Revista Brasileira de Cirurgia de Cabeça e Pescoço, 36, 146-151.

Justice, B. (1988). Stress, coping and health outcomes. In Russell, M.L. (1988). Stress management for chronic disease (pp. 14-30). Oxford, UK: Pergamon Press.

Karnell, L., Christensen, A. J., Rosenthal, E. L., Magnuson, J. S., \& Funk, G. F. (2006). Influence of social support on health-related quality of life outcomes in head and neck cancer. Head \& Neck, 29, 143-146.
Ledeboer, Q., Van Der Velden, L., De Boer, M., Feenstra, L., \& Pruyn, J. (2006). Palliative care for head and neck cancer patients in general practice. Acta Oto-Laryngologica, 126, 975980.

Leeuw, J. R. J., Graeff, A. D., Ros, W. J. G., Blijham, G. H., Hordijk, G. J., \& Winnubst, J. A. M. (2000). Prediction of depressive symptomatology alter treatment of head and neck cancer: The influence of pre-treatment physical and depressive symptoms, coping, and social support. Head \& Neck, 22, 799-807.

Llewellyn, C. D., Mcgurk, M., \& Weinman, J. (2005). Are psycho-social and behavioural factors related to health related-quality of life in patients with head and neck cancer? A systematic review. Oral Oncology, 41, 440-454.

Marmot, M., \& Wilkinson, R. (Eds.). (2006). Social determinants of health (2nd ed.). Oxford, UK: Oxford University Press.

McColl, M. A., Lei, H., \& Skinner, H. (1995). Structural relationschips between social support and coping. Social Science \& Medicine, 41, 395-407.

Medeiros, V. C. C., \& Peniche, A. C. G. (2006). A influência da ansiedade nas estratégias de enfrentamento utilizadas no período pré-operatório. Revista da Escola de Enfermagem da USP, 40, 86-92.

Meneses, M. P. R., \& Sarriera, J. C. (2005). Redes sociais na investigação psicossocial. Aletheia, 21, 53-67.

Nucci, N. A. G. (2003). Qualidade de vida e câncer: Um estudo compreensivo. Tese de Doutorado não-publicada, Faculdade de Filosofia, Ciências e Letras, Universidade de São Paulo, Ribeirão Preto, SP. 
Santana, J. J. R. A., Zanin, C. R. \& Maniglia, J. V. (2008). Câncer: enfrentamento e apoio social 383

Organização Mundial de Saúde. (2005). Classificação Internacional de Doenças para Oncologia (3a ed.). São Paulo: EdUSP.

Ostberg, V., \& Lennartsson, C. (2007). Getting by with a little help: The importance of various types of social support for health problems. Scandinavian Journal of Public Health, 35, 197-204.

Ribeiro, J. L. P., \& Rodrigues, A. P. (2004). Questões acerca do coping: A propósito do estudo de adaptação do brief cope. Psicologia, Saúde e Doenças, 5(1), 3-15.

Rosas, M., \& Baptista, F. (2002). Desenvolvimento de estratégias de intervenção psicológica para a cessação tabágica. Análise Psicológica, 20, 45-56.

Sartor, S. G., Eluf-Neto, J., Travier, N., Wünsch Filho, V., Arcuri, A. S. A., Kowalski, L. P., \& Boffetta, P. (2007). Riscos ocupacionais para o câncer de laringe: Um estudo de caso-controle. Cadernos de Saúde Pública, 23, 1473-1481.

Savoia, M. G. (1999). Escalas de eventos vitais e de estratégias de enfrentamento (coping). Revista de Psiquiatria Clínica, 26, 57-67.

Seeman, T. E. (1996). Social ties and health: The benefits of social integration. Annals of Epidemiology, 6, 442-451.

Seidl, E. M. F., Melchíades, A., Farias, V., \& Brito, A. (2007). Pessoas vivendo com HIV/AIDS: Variáveis associadas à adesão ao tratamento antiretroviral. Cadernos de Saúde Pública, 23, 2305-2316.

Seidl, E. M. F., Tróccoli, B. T., \& Zannon, C. M. L. C. (2001). Análise fatorial de uma medida de estratégias de enfrentamento. Psicologia: Teoria e Pesquisa, 17, 225-234.

Skinner, B. F. (2000). Ciência e comportamento humano (10a ed., R. Azzi \& J. C. Todorov, Trads.). São Paulo: Martins Fontes.
Trentini, M., Silva, S. H., Valle, M. L., \& Hammerschmidt, K. S. (2005). Enfrentamento de situações adversas e favoráveis por pessoas idosas em condições crônicas de saúde. Revista Latino-Americana de Enfermagem, $13,38-45$.

Valla, V. V. (1999). Educação popular, saúde comunitária e apoio social numa conjuntura de globalização. Cadernos de Saúde Pública, 15(Supl. 2), 7-14.

Van der Molen, B. (1999). Relating information needs to the cancer experience: 1 . Information as a key coping strategy. European Journal of Cancer Care, 8, 238-244.

Venturi, B. R. M., Pamplona, A. C. F., \& Cardoso, A. S. (2004). Carcinoma de células escamosas da cavidade oral em pacientes jovens e sua crescente incidência: Revisão de literatura. Revista Brasileira de Otorrinolaringologia, 70, 679-686.

Vidhubala, E., Latha, Ravikannan, R., Mani, C. S., \& Karthikesch, M. (2005). Doping preferentes of head and neck cancer patients: Indian context. Indian Journal of Cancer, 43, 6-11.

Zago, M. M. F., Sawada, N. O., Stopa, M. J. R., \& Martinez, E. L. (1998). O significado cultural de ser laringectomizado. Revista Brasileira de Cancerologia, 44, 139-145.

Zanin, C. R., Corrêa, C. G., Maniglia, J. V. (1999). Transtorno mental em pacientes com câncer de cabeça e pescoço submetidos à cirurgia e radioterapia e a radioterapia [Resumo]. Congreso Iberoamericano de Psicología Clínica y de la Salud: Avances Recientes em Psicologia Clínica y de la Salud, 1, 332.

Artigo recebido em 22/01/2008.

Aceito para publicação em 28/08/2008.

Trabalho derivado de monografia de finalização do Curso de Especialização e Aprimoramento em Psicologia da Saúde do Hospital de Base da Faculdade de Medicina de São José do Rio Preto-SP. 
384 Paidéia, 2008, 18(40), 371-384

Endereço para correspondência:

Jeanny Joana Rodrigues Alves de Santana. Rua Antônio Maia do Amaral, 560. CEP 38703-066. Patos de Minas-MG, Brasil.

Jeanny Joana Rodrigues Alves de Santana é especialista em Psicologia da Saúde pela Faculdade de Medicina de São José do Rio Preto-SP.

Carla Rodrigues Zanin é psicóloga da Faculdade de Medicina de São José do Rio Preto-SP.

José Victor Maniglia é médico do Hospital de Base da Faculdade de Medicina de São José do Rio Preto-SP. 